\title{
EFFECTS OF CELL SEEDING METHODS ON CHONDROGENIC DIFFERENTIATION OF RAT MESENCHYMAL STEM CELLS IN POLYHYDROXYBUTYRATE/CHITOSAN SCAFFOLDS
}

\author{
Giretová, M. ${ }^{1}$, Medvecký, L. ${ }^{1}$, Petrovová, E. ${ }^{2}$ \\ Čížková, D. ${ }^{2}$, Mudroňová, D. ${ }^{3}$, Danko, J. ${ }^{2}$ \\ ${ }^{1}$ Division of Functional and Hybrid Systems \\ Institute of Materials Research of the Slovak Academy of Sciences, Watsonova 47, Kosice \\ ${ }^{2}$ Institute of Anatomy, 3Institute of Immunology \\ University of Veterinary Medicine and Pharmacy in Kosice, Komenskeho 73, 04181 Kosice \\ Slovakia \\ mgiretova@saske.sk
}

\begin{abstract}
The aim of our study was to examine the effects of passive and active cell seeding techniques on in vitro chondrogenic differentiation of mesenchymal stem cells (MSC) isolated from rat bone marrow and seeded on porous biopolymer scaffolds based on polyhydroxybutyrate/chitosan (PCH) blends. This paper is focused on the distribution of the cells on and in the scaffolds, since it influences the uniformity of the created extracellular matrix (ECM), as well as the homogenity of the distribution of chondrogenic markers in vitro which ultimately affects the quality of the newly created tissue after in vivo implantation. The three types of cell-scaffold constructs were examined by: fluorescence microscopy, SEM, histology and quantitative analysis of the glycosaminoglycans after chondrogenic cultivation. The results demonstrated that the active cells seeded via the centrifugation of the cell suspension onto the scaffold guaranteed an even distribution of cells on the bulk of the scaffold and the uniform secretion of the ECM products by the differentiated cells.
\end{abstract}

Key words: bone marrow; glycosaminoglycans; ECM; mesenchymal stem cells; polyhydroxybutyrate/chitosan scaffold

\section{INTRODUCTION}

The healing process of injured cartilage is insufficient due to the fact, that cartilage is an avascular and aneural tissue with a low number of chondrocytes which results in osteoarthritic changes and the production of inferior fibrocartilage. Several techniques designed to restore injured articular cartilage like: e.g. autologous chondrocyte implantation, microfracture, mosaikoplasty, are known in medicine [26]. At the present time, much attention is paid to the field of regenerative medicine and cartilage tissue engineering (TE), where with the appropriate cell type, suitable scaffold for cell seeding and biological factors or substances, which control the cell differentiation into desired lineages are studied. The key role of the scaffold is to support cell colonization, migration, growth, differentiation, and the development and integration of formed tis- 
sue [27]. Scaffolds for in vitro chondrogenesis has been characterized as biomaterials based on biopolymers; e.g. collagens, polyhydroxyalkanoate, hyaluronate alginate, and polyurethanes $[3,4,10,14]$. Chitosan is a natural aminopolysaccharide consisting of sugars close to natural glycosaminoglycans (GAGs) characteristic for cartilage tissue. Chitosan is formed by the alkaline deacetylation of chitin-the second most abundant natural polysaccharide in the world. Another benefit for the utilization of chitosan includes its antimicrobial properties [11]. M a dih ally and $\mathrm{M}$ a t $\mathrm{th}$ e w studied animal tissue tolerance to chitosan based implants with the conclusion, that this type of material causes a minimal body response and is considered as biocompatible [19]. These implants were degraded hydrolytically with lysozymes and the rate of degradation was inversely proportional to the degree of crystallinity. $\mathrm{Y}$ a $\mathrm{m}$ a $\mathrm{n}$ e et al. compared the in vitro properties of a hybrid composite consisting of hyaluronic acid (HA) coated chitosan fibers and the properties of pure chitosan [34]. Cell adhesion, proliferation and aggrecan synthesis were significantly higher in the hybrid composite with HA than in chitosan. SEM observations showed a typical chondrogenic phenotype of cells with a lot of extracellular matrix. Immunohistochemical staining has demonstrated the rich production of collagen type II by chondyrocytes.

$\mathrm{Cho}$ et al. demonstrated the ability to differentiate MSC into chondrocytes using an injectable gel based on chitosan-Poly-N-isopropyl acrylamide [15]. MSCs were cultured in vitro and after the injection of the cell-gel complex into the animal organism the cartilage tissue formation was revealed.

$\mathrm{T}$ a $\mathrm{n}$ et al. studied hydrogels consisting of N-succinyl chitosan and aldehyde hyaluronate with encapsulated bovine chondrocytes [29]. The hydrogel allowed the survival of chondrocytes and the maintenance of their typical phenotype. The authors concluded that the composite system has the potential for tissue engineering applications.

$\mathrm{Ch}$ e $\mathrm{n}$ et al. manufactured three-dimensional substrates consisting of chondroitin sulphate (CS), dermatan sulfate (DS) and chitosan in various formulations with potential use in cartilage tissue engineering [13]. The addition of CS and DS positively affected the cell morphology, glycosaminoglycan and collagen production as well as expression of the corresponding genes. W a $\mathrm{ng}$ et al. implanted a 3D substrate consisting of poly (3-hydroxybutyrate (PHB) and 3-hydroxyhexanoate) seeded with rabbit chondrocytes into rabbits after 10 days of in vitro culture [33]. The treated defects in rabbits were filled with cartilage tissue with good connection with the subchondral bone. The scaffolds showed higher accumulation of ECM with Type II collagen and GAGs.

Mesenchymal stem cells (MSCs) are used as a cell source for TE and specifically in cartilage regeneration due to their relatively simple availability from multiple tissues (bone marrow, hair follicles, dental pulp, adipose tissue), high proliferation capacity in laboratory conditions and the ability to differentiate among other cell types (osteocyte, adipocyte) including chondrocytes $[1,6,31]$. Their main tasks in chondrogenic differentiation of MSC are affecting and control of the differentiation process from the point of view of enhancing the synthesis of collagen II, aggrecan and GAGs by differentiated cartilage cells. The effective biological active molecules responsible for the in vitro differentiation of MSC into the chondrogenic lineage are dexamethasone and transforming growth factor as supplements in chondrogenic differentiation culture media $[8,28]$.

For successful tissue regeneration using cartilage tissue engineering, it is recommended that the optimal pore size of scaffolds be between $100-300 \mu \mathrm{m}$. A critical step involves the cell seeding on to the porous scaffold [22]. The passive seeding technique is based on dropping cells onto the scaffold surface followed by the cell infiltration through the scaffold microstructure. On the other hand, the active cell seeding utilises a certain external factor, which improves the penetration of cells into the interior of the substrate (rotation, centrifugation, magnetic field) and the results have demonstrated improved cell distribution and ECM formation by differentiated cells by this method [2, $9,30]$.

The aim of this study was to examine the effects of passive and active cell seeding techniques on the in vitro chondrogenic differentiation of mesenchymal stem cells isolated from rat bone marrow and seeded on porous biopolymer scaffolds based on polyhydroxybutyrate/chitosan (PCH) blends.

\section{MATERIALS AND METHODS}

\section{Scaffold preparation}

Porous biopolymer polyhydroxybutyrate/chitosan (PCH) scaffolds were prepared according to $\mathrm{M}$ e d v e c k y 
et al. [21]. The PCH scaffolds with the PHB:Chit ratio equal to $1: 1$ were prepared by the precipitation of $\mathrm{PHB}(\mathrm{PHB}$, GoodFellow, dissolved in propylene carbonate) and chitosan (Chit, SigmaAldrich, dissolved in $1 \%$ acetic acid) mixture. After stirring for 10 minutes, acetone was added to the slurry until complete precipitation of the biopolymers occurred. The resulting polymer blends were washed with distilled water, filtered, molded in molds (scaffold type A, $\mathrm{B}$-discs) or microcentrifuge tubes (scaffold type $\mathrm{C}$-cone) and frozen at $-20^{\circ} \mathrm{C}$. Finally samples were lyophilized (Ilshin) for 6 hours and sterilized in an autoclave. The microstructure of the scaffold was modified by changing the water content in the suspension.

The microstructure of the scaffolds was observed by the scanning electron microscopy (FE SEM JEOL7000) after the deposition of the conductive carbon layer on the scaffold surface. The distribution of the molecular weights of PHB and chitosan in the mixtures was determined by gel permeation chromatography (GPC, Watrex, RI detector). Due to the high porosity of the scaffolds, they were free of closed pores and the true density of the PCH scaffolds was determined by Helium Pycnometer (AccuPyc II, Micrometics). The porosity of the scaffolds (\%) was calculated from the true density of the blend, mass and dimensions of the individual scaffold.

\section{Isolation and culture of rat MSCs}

The bone marrow was isolated from the long bones (femur, tibia) of an adult male Wistar rats (300 g) cadavers (for up to 3 hours at $4{ }^{\circ} \mathrm{C}$ ). The full bone marrow was flushed with ice-cold DMEM LG (Dullbecco's Modified Essential Medium; low glucose, Sigma-Aldrich, UK) $+10 \%$ FBS (fetal bovine serum, Biowest, France) culture medium, homogenized, and centrifuged at $200 \mathrm{~g}$ for $10 \mathrm{~min}$. The cell pellet was plated on a $75 \mathrm{~cm}^{2}$ culture flask (SPLLife Sciences, Korea) and cultured in $15 \mathrm{ml}$ of DMEM LG with $10 \%$ FBS, and $1 \%$ penicillin-streptomycin-amphotericine (ATB-ATM solution, Sigma-Aldrich, UK); and incubated at $37{ }^{\circ} \mathrm{C}$ in a humidified atmosphere with $5 \% \mathrm{CO}_{2}$. Nonadherent cells were removed by changing the medium after 48 h. The cells were passaged upon reaching $90 \%$ of confluence. The subconfluent cell layer from passage 2 were released and cells were used for the determination of the MSC multidifferentiation capacity, confirmation of MSC surface markers (CD29, CD90, CD45) by flow cytometry and in vitro chondrogenesis experiments. The cells were seeded in biopolymeric scaffolds by simple dropping of the cell suspension on to the surface of the scaffold and infiltrated the cells to the porous structure of the scaffold by gentle centrifugation.

\section{Phenotypic characterization of MSC by flow cytometry}

For flow cytometric analysis of the cells, direct immunofluorescence staining was used, with a combination of conjugated monoclonal antibodies: CD45/CD29/CD90.1 (eBioscience, USA). The specification of the antibodies used is presented in Table 1 .

The flow cytometric analysis was performed on a six color BD FACSCantoTM flow cytometer equipped with blue $(488 \mathrm{~nm})$ and red $(633 \mathrm{~nm})$ lasers (Becton Dickinson Biosciences, USA). The data were analyzed using the $\mathrm{BD}$ FACS DivaTM software. The proportions of cells expressing analyzed CD markers were expressed in percentages.

\section{Multidifferentiation capacity of MSC}

The multidifferentiation ability of isolated adherent cells was confirmed by a commercially purchased kit StemPro Chondrogenesis, Adipogenesis and Osteogenesis Differentiation Kit (Gibco) according to the manufacturer instructions. The cells were cultured in the differentiation media for up to 21 days; the medium was changed three times a week. The differentiated cells were fixed with $4 \%$ formaldehyde and stained-fat vacuoles of adipocytes with Oil Red (Sigma), calcium deposits produced by osteoblasts with Alizarin Red S (Sigma) and GAGs in micromasses of chondrocytic cells with Alcian Blue (Sigma).

Table 1. Specification of the anti-mouse monoclonal antibodies used

\begin{tabular}{cccccc}
\hline Type & Fluorochrome & Clone & Isotype & Concentration & Volume $/ 10^{5}$ cells \\
\hline anti-CD45 & APC & OX1 & $\operatorname{lgG1,k}$ & $0.2 \mathrm{mg}^{\mathrm{ml}} \mathrm{l}^{-1}$ & $2.5 \mu \mathrm{l}$ \\
anti-CD29 & R-PE & HMb1-1 & $\operatorname{lgG}$ & $0.2{\mathrm{mg} \cdot \mathrm{ml}^{-1}}^{-1}$ & $5 \mu \mathrm{l}$ \\
anti-CD90.1 & FITC & DX5 & $\operatorname{lgG} 2, \mathrm{~K}$ & $0.5 \mathrm{mg} \cdot \mathrm{ml}^{-1}$ & $0.25 \mu \mathrm{l}$ \\
\hline
\end{tabular}




\section{Cell seeding into the biopolymeric scaffolds}

The MSC were enzymatically released from the culture flasks, counted and the concentration of the cells in suspension was adjusted. The final scaffold characteristics (size and volume), cell concentration as well as method of the cell seeding are described in Table 2. Scaffolds were after seeding transferred separately into wells of 48 nonadherent culture plate (Greiner Bio-One) and incubated $\left(37^{\circ} \mathrm{C}, 5 \%\right.$ $\mathrm{CO}_{2}, 95 \%$ humidity) for $2 \mathrm{~h}$. Following the $0.5 \mathrm{ml}$ of complete chondrogenic medium (DMEM HG-high glucose 4.5 g. $\left.1^{-1}\right), 1 \%$ ITS + $3.50 \mu \mathrm{g} \cdot \mathrm{ml}^{-1}$ ascorbic acid, $40 \mu \mathrm{g} \cdot \mathrm{ml}^{-1}$ proline, 10 ng.ml ${ }^{-1}$ TGF b1, $1 \%$ HEPES, $1 \%$ NEAA, $1 \%$ ATB-ATM solution (all from Sigma) was added to each well containing the cell-scaffold construct. The medium was changed three times a week.

\section{Morphology and topography of MSC seeded in scaffolds by fluorescence staining}

Live/dead staining (fluorescein diacetate/ propidium iodide), acridine orange and DAPI(4,6-diamidino-2-phenylindole) staining were used for the visualisation of the cells focused on their morphology, density and topography on the scaffold surfaces so as to evaluate the cross-sections after 2 and 4 weeks of chondrogenic cultivation.

Fluorescein diacetate is metabolised by live cells to a fluorescent product and stains the living cells green. Propidium iodide is permeable by damaged cell membranes and stains the dead cells red (live/dead staining). DAPI stains the cell nuclei blue. Acridin orange stains the cell nuclei yellow-green to orange-red. After washing with PBS, the stained cell-scaffold constructs were observed by a fluorescence optical microscope Leica DM IL LED, blue filter.

For obtaining quantitative data, which could more precisely characterize the distribution of cells across the substrate, the the conical scaffold $\mathrm{C}$ was sectioned into 3 parts: surface, middle and bottom part. The thickness of each part was about 2 milimeters and the cell nuclei on captured images stained with DAPI were counted on areas of $1 \mathrm{~mm} 2$.

\section{Histological staining of cell-scaffold constructs}

After 4 weeks of cultivation in complete chondrogenic media, the constructs were removed, washed and fixed in $4 \%$ paraformaldehyde. The specimens were sectioned (Leica RM 2255) in 5-10 $\mu$ m slides and stained with alcian blue for 30 minutes (GAGs staining); the cell nuclei were stained with nuclear fast red. Scaffolds $C$ failed to prepare for histological staining due to their disruption in paraffin.

\section{Scanning electron microscopy staining of cells- scaffold constructs}

After 2 and 4 weeks of cultivation of the cell-scaffold constructs in chondrogenic media, scaffolds were removed, washed with PBS and fixed in $2.5 \%$ glutaraldehyde in PBS for 24 hours at $4{ }^{\circ} \mathrm{C}$. After dehydratation in an ethanol gradient from 30 to $100 \%$ and freeze drying (Illshin), the specimens were sputter-coated with carbon and observed (JEOL FM SEM JSM-7000F).

\section{Determination of DNA and GAG-s content in cell-scaffold constructs}

After 4 weeks of cultivation in chondrogenic medium, scaffolds were removed and washed with PBS and lysed in papain buffer after homogenization (Tissuerupter, Quagen) at $60^{\circ} \mathrm{C}$ for 24 hours. The aliquots of supernatants were used for GAGs determination by the DMMB methodology and DNA estimation by Hoechst 33258. To determine the GAGs content, the $250 \mu$ solution of the dimethylmethylene blue (DMMB) was added to $50 \mu$ of the supernatant and absorbance at 450 and $525 \mathrm{~nm}$ was measured by UV VIS spectrophotometer (Shimadzu, UV-1800). The

Table 2. Scaffold characteristics, cell density on scaffolds and methods of cell seeding

\begin{tabular}{|c|c|c|c|c|c|c|}
\hline Type & Composition & $\begin{array}{l}\text { Cell seeding } \\
\text { method }\end{array}$ & $\begin{array}{l}\text { Average scaffold } \\
\text { porosity* }\end{array}$ & $\begin{array}{l}\text { Scaffold volume } \\
(\mu \mathrm{l})^{*}\end{array}$ & Cells/scaffold & $\begin{array}{c}\text { Cells.cm-3 scaf- } \\
\text { fold }\end{array}$ \\
\hline$A$ & $\mathrm{PCH}(1: 1)$ & dropping & $85 \pm 4.1$ & $50 \pm 5$ & $3.5 \times 10^{5}$ & $7.0 \times 10^{6}$ \\
\hline B & chitosan (100\%) & dropping & $92 \pm 4.8$ & $50 \pm 5$ & $3.5 \times 10^{5}$ & $7.0 \times 10^{6}$ \\
\hline C & $\mathrm{PCH}(1: 1)$ & $\begin{array}{l}\text { centrifugation } \\
\text { (1400 rpm/5 min) }\end{array}$ & $94 \pm 3.8$ & $100 \pm 15$ & $3.5 \times 10^{5}$ & $3.5 \times 10^{6}$ \\
\hline
\end{tabular}




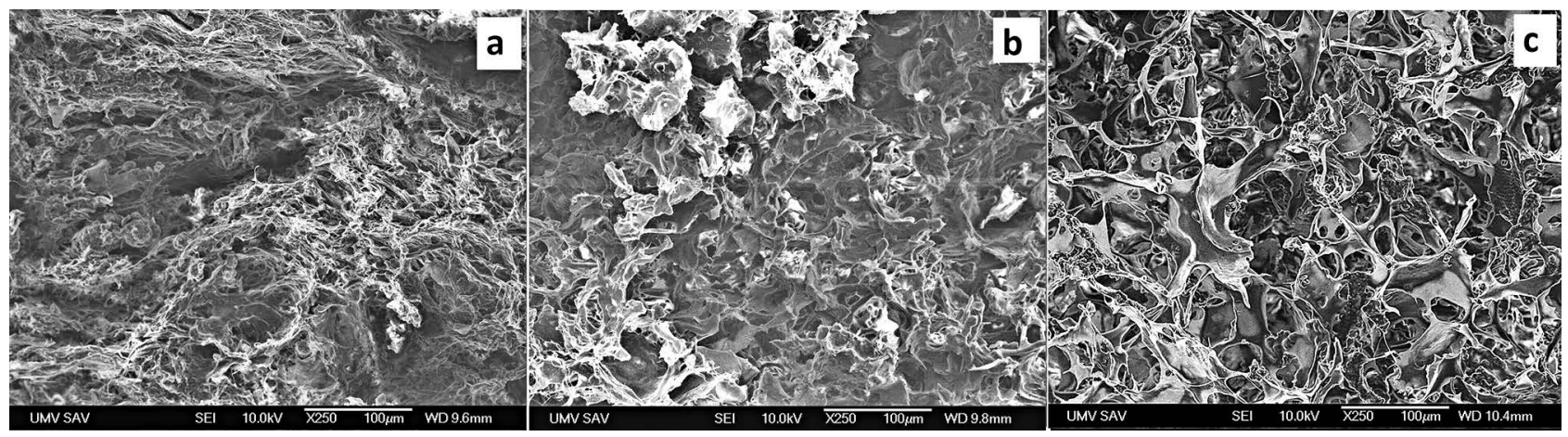

Fig. 1. SEM images of scaffold microstructures: a) A; b) B; c) C-type

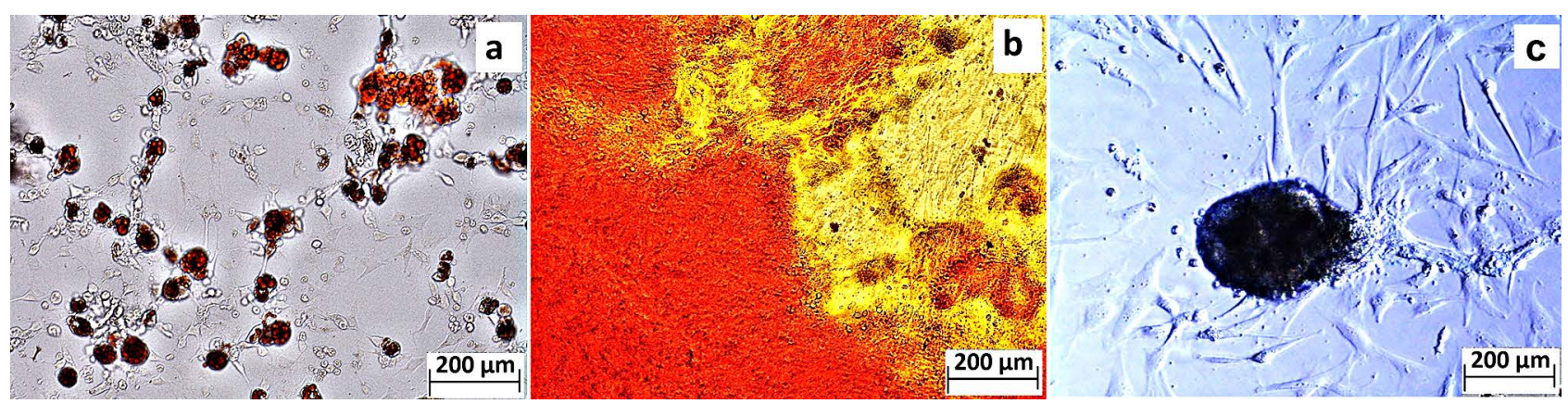

Fig. 2. Multidifferentiation ability of MSC:

b) adipogenic differentiation, oil red staining of fat vacuoles; c) osteogenic differentiation, alizarin red staining of calcium deposits;d) chondrogenic differentiation, alcian blue staining of GAGs

chondroitin sulfate from shark cartilage (Sigma) was used as a standard for GAG calibration.

The DNA content in cell lysate from cell constructs was determined using Hoechst 33258. The $20 \mu \mathrm{l}$ of supernatant was added to Hoechst 33258 buffer solution $(180 \mu \mathrm{l})$ and the DNA content was determined from the calibration curve by fluorimetry (Picofluor, Turner biosystems). The DNA from a calf thymus (Sigma) was used as a standard.

All of the quantitative measurements were performed on cell-scaffold constructs $(n=3)$ and then statistically evaluated by ANOVA (Statmost32 statistical programme). The statistical significance of results was determined by one- and two-way ANOVA ( $\mathrm{P} \otimes 0.05)$.

\section{RESULTS}

\section{Scaffold characterization}

The highly porous spongy-like microstructure of the scaffolds were obtained after lyophilization (Fig. 1). The images document a heterogeneous open microstructure with a high proportion of more regular macropores up to $100 \mu \mathrm{m}$ size with mutual interconnection via smaller spherical pores of $<40 \mu \mathrm{m}$. This microstructure allows a faster diffusion of media into the interior of the scaffolds after cell seeding. Also a dense network of fine spherical micropores (diameter $<10 \mu \mathrm{m}$ ) were observable in the pore walls of the scaffolds (Fig. 1b, c). The calculated porosities of the scaffolds are listed in Table 2 and all of the scaffolds achieved the $\geq 85 \%$ level. The gel permeation chromatography (GPC) analysis showed that the average molecular mass $(\mathrm{Mw})$ of Chit and PHB in the mixtures were $41 \mathrm{kDa}$ and $80 \mathrm{kDa}$ respectively.

\section{Multidifferentiation capacity and phenotype characterization of MSC}

The flow cytometric analysis confirmed that $>95 \%$ of the cells expressed CD90, CD29 and around $1.1 \%$ of the cell population expressed CD45. The isolated cells were able to differentiate to adipocytes: red fat vacuoles in adipocytes stained with oil red (Fig. 2a); osteoblasts: red colored calcium deposits stained with alizarin red (Fig. 2b); and chondrocytes: blue stained GAGs in micromasses stained 

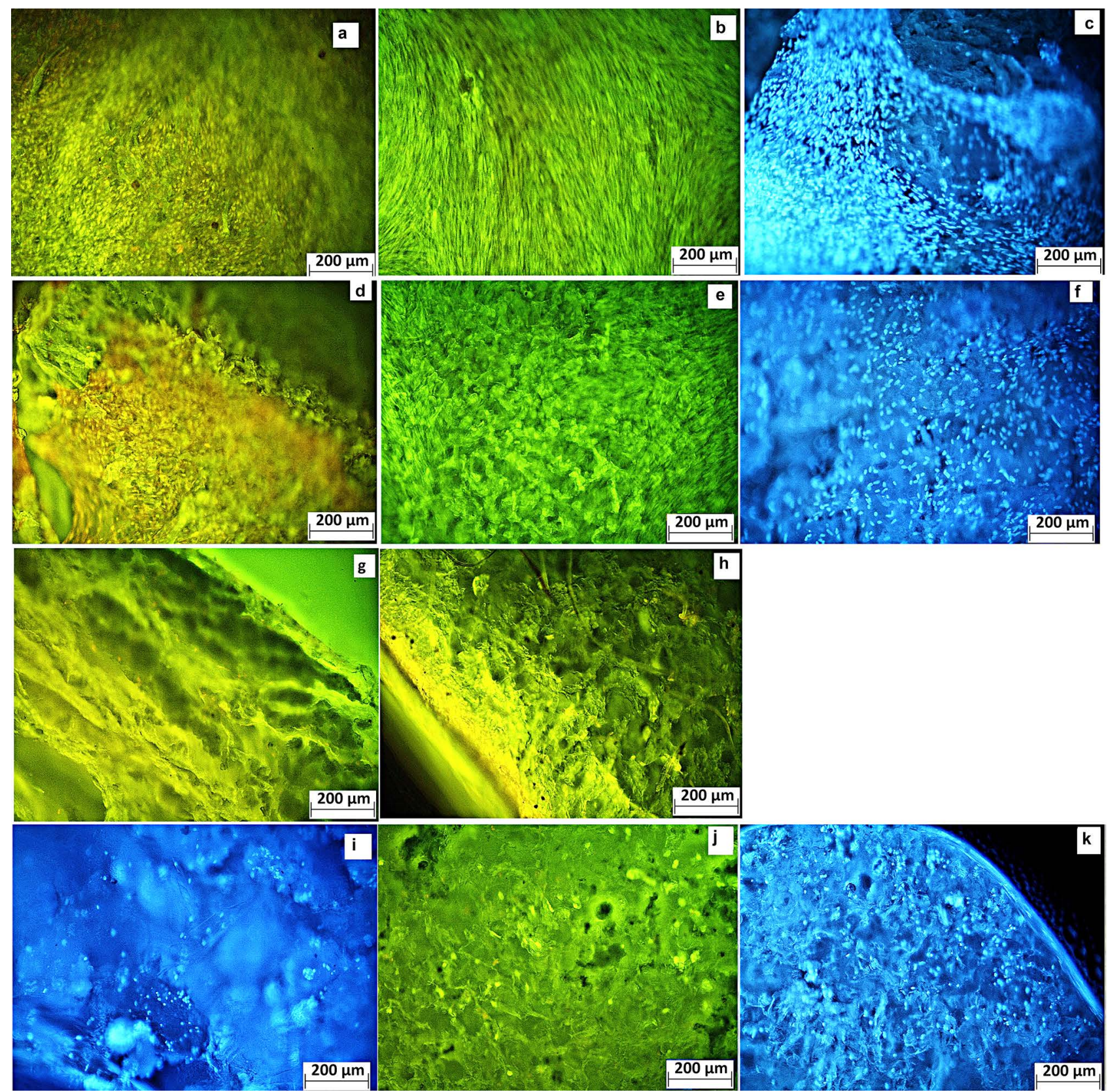

Fig. 3. Distribution of cells on scaffolds characterized by fluorescence microscopy:

A-type-surface after 2 (a-acridine orange) and 4 weeks (b-live/dead; c- DAPI) of chondrogenic cultivation; cross-sections after 4 weeks of culture (g-acridine orange); B-type-surface after 2 (d-acridine orange) and 4 weeks (e-live/dead; f- DAPI) of chondrogenic cultivation; cross-sections after 4 weeks of culture (h-live/dead); C-type- cross-sections after 2 (i-DAPI) and 4 weeks (j- live/dead; k- DAPI) of chondrogenic cultivation

with alcian blue (Fig. 2c). These facts confirmed that cells isolated from rat bone marrow were MSC's.

\section{Morphology and topography of MSC seeded in scaffolds by fluorescence staining}

Figs. 3a, b, c, d, e, and $\mathrm{f}$ showed cell distributions on A and B scaffolds after 2 and 4 weeks of MSC cultivation in the chondrogenic media. An enormously dense cell population, adhered to the surface was revealed on the sur- face of the scaffolds. Multiple cell layers with extracellular matrix production were identified using the fluorescence staining techniques. The cross-section of the scaffolds confirmed the negligible penetration of cells into the inner porous structure, as shown in Fig. 3g and h. A layer of living cells is clearly visible on the scaffold surfaces. Also the live/dead staining (Figs. 3b, e) did not show the presence of dead cells on the scaffolds, which confirms the low cytotoxicity of the biocomposites. 


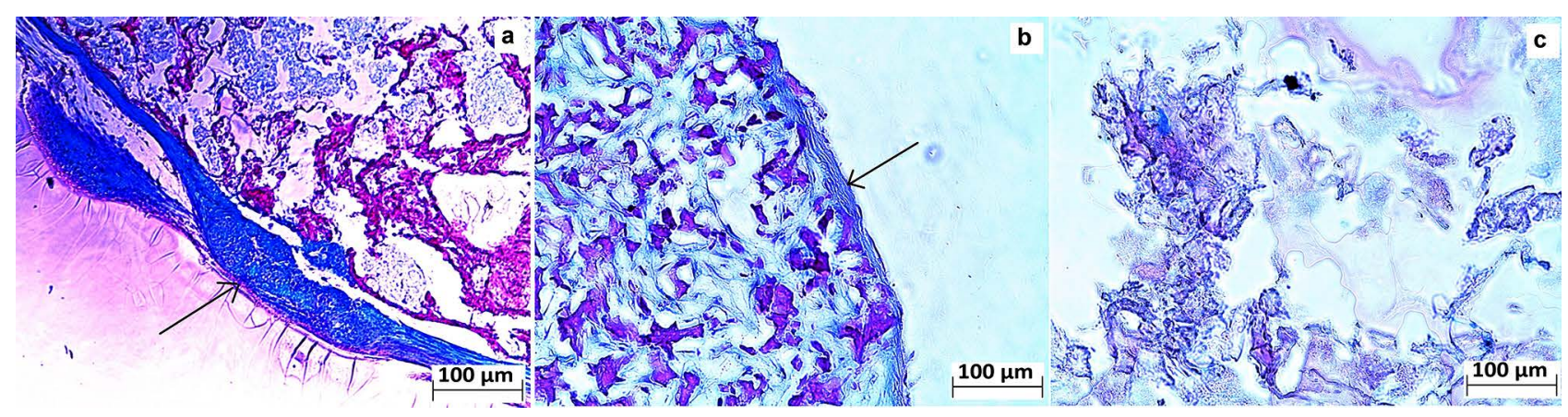

Fig. 4. Histological staining with alcian blue of scaffold A (a) and B (b) after 4 weeks of chondrogenic cultivation and scaffold A without cells (c). Arrows show layer of cells producing cartilage like tissue (positive GAG-s)

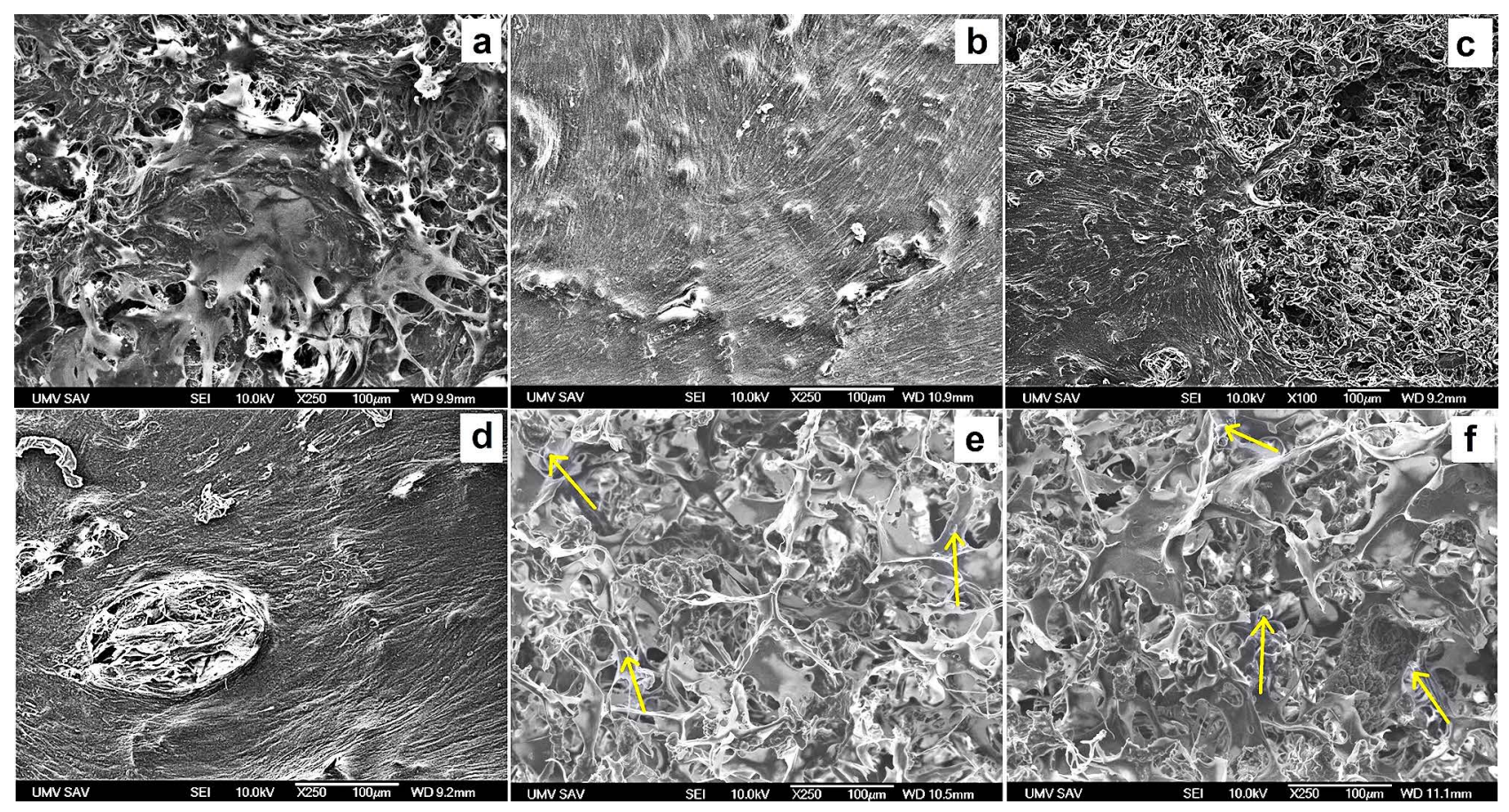

Fig. 5. Surface microstructure of scaffolds A (a, b), B (c, d) and cross-section of scaffold C (e, f). Arrows indicate adhered cells) after 2 weeks and 4 weeks of chondrogenic cultivation

In Figs. 3i, j, and k; a uniform distribution of cells is observable on the surfaces and on the cross-sections of scaffolds $\mathrm{C}$, but the density of cells was lower than on the A or B samples. The acridine orange staining of scaffolds $C$ was qualitatively unsatisfactory.

The average amount of cells on each assessed part of the scaffold C was $1470 \pm 180$ cells. $\mathrm{mm}^{-2}$. On the surface part, there were $1320 \pm 200$ cells. $\mathrm{mm}^{-2}$; on the middle part and bottom parts there were $1180 \pm 270$ cells. $\mathrm{mm}^{-2}$. Based on these findings it can be stated that cells were randomly distributed and the number of cells in each part of the scaffold $\mathrm{C}$ was not statistically significantly different $(\mathrm{P}<0.05)$.

\section{Histological staining of cell-scaffold constructs}

Figs. $4 \mathrm{a}$ and $\mathrm{b}$ revealed the histological staining of A and B type scaffolds by Alcian Blue, which demonstrated the presence of GAGs. The arrows indicate cell layers on the surface producing a cartilage-like tissue positively staining dark blue by Alcian blue. The scaffold cross section without seeded cells characterized by porous structure with polymer fibers created pore walls is demonstrated in Fig. 4c. Note, the partial staining of biopolymer blends (chitosan) can be visible in Fig. 4, but GAGs were stained more intensely blue in the multilayers of cells. 
Table 3. Measured content of GAGs and DNA in cell-scaffold constructs

\begin{tabular}{cccc}
\hline Scaffold & Seeding of MSC & $\begin{array}{c}\text { GAG-s content } \\
(\mathbf{n g} / \mathbf{s c a f f o l d}) *\end{array}$ & $\begin{array}{c}\text { DNA content } \\
(\boldsymbol{\mu g} / \mathbf{s c a f f o l d}) *\end{array}$ \\
\hline A & dropping & $130 \pm 30$ & $0.903 \pm 0.1$ \\
B & dropping & $220 \pm 30$ & $0.609 \pm 0.09$ \\
C & centrifugation & $75 \pm 8$ & $0.944 \pm 0.1$ \\
\hline \multicolumn{4}{c}{$*$ - mean \pm standard deviation }
\end{tabular}

\section{SEM evaluation of cells-scaffold constructs}

The SEM images of A and B scaffold surfaces after 2 and 4 weeks of MSC cultivation are shown in Figs. 5a, b, $c$, and $\mathrm{d}$. The images are consistent with the observations from the fluorescence microscopy and histological staining. The scaffold surfaces are almost completely covered with cell multilayers and clearly indicate the excellent adherence of the cells and layers to the scaffold surface. These facts verify a low cytotoxicity of PCH scaffolds.

In the case of the scaffold type C, the SEM showed the microstructure with a cell population adhered and distributed on individual pore walls with partial filling of the scaffold pores. The individual cells are relatively difficult to distinguish from the highly porous, plate-like interconnected microstructure of the biopolymers (Figs. 5e and f).

\section{Determination of DNA and GAG-s content in cell-scaffold constructs}

The average GAG contents determined by DMMB after 4 weeks of culture of each cell-scaffold type construct are shown in Table 3. We observed statistically significant differences in GAG contents $(\mathrm{P}<0.05)$ between the individual sample types A, B and C with the higher GAG content in the B substrate $(220 \pm 30 \mathrm{ng})$ and much lower in the $C$ type substrate (75 $\pm 10 \mathrm{ng}$ ).

Table 3 shows also the amount of DNA in cell-scaffold constructs after chondrogenic cultivation using Hoechst 33258. Similar amounts of DNA were found in the A and C scaffolds, whereas a statistically significant decrease was revealed in scaffold $\mathrm{B}(\mathrm{P}<0.05)$.

\section{DISCUSSION}

The isolated MSC's from rat bone marrow represent an adherent population of spindle-shaped fibroblast-like cells, which were able to differentiate into bone, cartilage and fat cell lineages during cultivation under defined conditions. During the in vitro chondrogenic differentiation of MSCs seeded on scaffolds, the supplementation of the culture media with growth factors and other active biological substances is strongly recommended. Certain biologically active molecules which bind to the MSC receptors affects both the entire differentiation process and the synthesis of extracellular matrix components by the cells. In the chondrogenic differentiation of MSC, glucocorticoid-dexamethasone and growth factor TGFb1 played crucial roles [28]. The chondrogenic differentiation was mostly carried out in a culture medium without FBS (FBS is a mixture of biological agents like growth factors, hormones, etc. which can adversely affect the behavior of the cells in the differentiation process) and FBS was replaced by the supplement containing insulin, transferrin, selenite, linoleic acid, oily acid and bovine serum albumin (commercial product ITS +3$)$, which is used as a serum replacement in serum-free cell cultivation during the chondrogenic differentiation of the stem cells. Also, one of the most used culture media is DMEM HG. DMEM HG contains up to $4.5 \mathrm{~g}$ of glucose per liter of medium and enhanced glucose amount is very important in the cultivation of an enormously high number of MSCs because glucose serves as a source of readily available energy for the cells [16, 28, 32]. In our experimental work, the isolation and expansion of MSC took place in DMEM LG medium with the addition of $10 \%$ FBS. However, MSC differentiation to chondrocytes (4 weeks) was performed in a culture medium of DMEM HG with chondrogenic supplements and successful cell differentiation was experimentally demonstrated.

The 3D-porous scaffolds must meet the requirements of biocompatibility, bioresorbability or biodegradability, good mechanical strength, shape, interconnected pores of appro- 
priate size distribution to allow the cells to penetration into the scaffolds as well as allow the flow of nutrients and metabolite between the culture medium and the cells $[10,18$, 24,]. The above conditions fulfill biomaterials of polymer origin, typically collagen, hyaluronic acid, and chondroitin sulphate in the case of cartilage regeneration $[7,12,14$, 25]. A number of authors have developed chitosan-based scaffolds and their composites with other polymers or inorganic compounds (e.g. calcium phosphates). As is well known, chitosan is characterized by favorable properties which predispose its use as a cell scaffold, especially in the regeneration of articular cartilage. Glucosamine groups of chitosan are structurally similar to the GAGs of the cartilage extracellular matrix and positively affect chondrocyte differentiation. In the living organism, it can be partially enzymatically degraded with lysozymes [5, 20, 23, 35]. The cost of chitosan is several times lower than collagen and hyaluronate which is a prerequisite to its wider availability for patients with traumatic cartilage damage. The porous interconnected structure of the polymer scaffolds provides a large area for the proper distribution, adhesion and cell proliferations as for neovascularization of the scaffold [17, $22]$. The pore size of the polymer scaffolds macropores prepared in our work was in the range of $50-100 \mu \mathrm{m}$ with interconnection via micropores of $<30 \mu \mathrm{m}$ diameter. The open pore spongy-like microstructure significantly improves the nutrients inflow to the deeper parts of the scaffold. A critical step in the cartilage tissue engineering is the cell seeding on the porous scaffold and our results are comparable with results of other research groups [2, 30]. The most appropriate method ensuring the uniform distribution of cells to the inner scaffold pores was active seeding via introducing the cell suspension to the scaffold by centrifugation. In the second method based on dropping the cell suspension on to the scaffold surface, the cells didn't penetrate into the pores and extracellular matrix formation was formed only on the scaffold surface. The cell seeding with very high concentrations for the in vitro chondrogenesis is parallel to cell condensation during embryonic development of cartilage in a living organism. For induction of MSC into a chondrogenic lineage, tight contacts between cells are desirable and the recommended cell concentrations applied on the scaffold is between 5 and 10 million cells per $\mathrm{cm} 3$ of porous scaffold. The much higher cell concentrations as described above were even applied for successful chondrogenesis [2]. In the case of PCH scaf- folds, the applied concentrations of MSC varied depending on the method of cell seeding. The cell concentrations used in dropping method met the requirement for cell seeding density on the porous cell scaffold contrary to this one in the centrifugation method with the lower cell density. The PCH scaffolds showed the suitable properties for chondrogenic differentiation of MSC's with production typical of the extracellular matrix component of hyaline-like cartilage-GAGs.

For comparison in Table 3, the amount of DNA in the cell-scaffold constructs of $\mathrm{A}$ and $\mathrm{C}$ was approximately the same $(0.9 \mu \mathrm{g}$ DNA/scaffold $)$ contrary to the B type samples where about a $30 \%$ lower amount was found. The dense multilayers of cells were formed after culture on scaffolds A and B (Figs. 3a-f; 4a, b; 5a-d) and such a cell arrangement does not allow for further cell proliferation on scaffolds because it is not of sufficient size for another adhesion and spreading of cells. Simply said, the confluence was achieved. On the other hand, a 3D macroporous microstructure with large areas of pore walls in the scaffold $\mathrm{C}$ firstly makes it possible to obtain more homogeneous and uniform distribution of cells in the scaffold volume (Figs. 3i, j, and k; and 5e, and f), but the density of the adhered cells in the scaffold was not high enough for sufficient mutual cell interconnections during the short-time period after seeding which resulted in lower production of GAGs by the cells on these samples. In the case of scaffolds type A and B, the amount of produced GAGs was around 3 times higher than in the case of substrate C. We concluded, that the abundant production of GAGs by cells in the A and B scaffolds was related to the fact, that the cells formed a multilayer on the scaffold surface and the contact between the cells with each other was very tight (parallel to the culture of the chondrogenic pellets or micromasses formed by the very concentrated population of MSC), and this fact supported the differentiation of the cells in multilayers with good GAGs production. It is possible to assume that the prolonged cultivation of MSC's can enhance the cell population with significant effects on the production of cartilage markers. Based on recent papers, it can be assumed that the integrity of the constructs with active cells evenly distributed therein will be more satisfactory compared to scaffolds with active cells adhered to outer scaffold surface only and following overgrown with a differentiated cell layer on its surface and partially ingrowth tissue after implantation. However, it is necessary to consider the cur- 
rent health condition of the patient with injured cartilage, the cartilage lesion position relative to the anatomy of the entire joint, lesion size, its thickness, and the surgery technique used.

In our future research work, we will focus on increasing the initial cell seeding concentration and improving the active cell seeding techniques into porous scaffolds of polymeric origin.

\section{CONCLUSIONS}

Two different methods of seeding cells into biopolymer scaffolds were compared. The synthesized biopolymer scaffolds were not cytotoxic; the cells grew and differentiated on scaffold surfaces and in scaffold pores. The cells seeded by dropping on scaffolds $\mathrm{A}$ and $\mathrm{B}$ produced higher amounts of GAGs in comparison to cells seeded by centrifugation into scaffold C. For chondrogenic cultivation and GAGs production by differentiated cells seeded in suitable porous scaffolds, there is a key prerequisite that a high enough cell seeding concentration and selecting the right cell seeding techniques be utilized. The developed porous PCH scaffold could be utilized in cartilage tissue engineering in veterinary and human medicine due to its satisfactory properties and low cost of input materials for scaffold synthesis.

\section{ACKNOWLEDGEMENTS}

This study was supported by the Slovak Grant Agency of the Ministry of Education of the Slovak Republic and the Slovak Academy of Sciences, Project No. 2/0047/17.

\section{REFERENCES}

1. Barry, F. P., Murphy, J. M., 2004: Review Mesenchymal stem cells: clinical applications and biological characterization. Int. J. Biochem. Cell Biol, 36, 568-584. DOI: 10.1016/j.biocel.2003.11.001.

2. Bornes, T. D., Jomha, N. M., Mulet-Sierra, A., Adesida, A. B., 2016: Optimal seeding densities for in vitro chondrogenesis of two- and three-dimensional-isolated and expanded bone marrow-derived mesenchymal stromal stem cells within a porous collagen scaffold. Tissue Engn. C: Methods, 22, 208220. DOI: 10.1089/ten.tec.2015.0365 58

3. Collins, M. N., Birkinshaw, C., 2013: Hyaluronic acid based scaffolds for tissue engineering-A review. Carb. Pol., 92 (2), 1262-1279. DOI 10.1016/j.carbpol.2012.10.028.

4. Deng, Y., Lin, X. S., Zheng. Z., Deng. J. G., Chen, J. CH., Ma, H., Chen, G. Q., 2003: Poly (hydroxybutyrate-co-hydroxyhexanoate) promoted production of extracellular matrix of articular cartilage chondrocytes in vitro. Biomaterials, $24,4273-4281$

5. Deng, CH. M., He, L. Z., Zhao, M., Yang, D., Liu, Y. 2007: Biological properties of the chitosan-gelatin sponge wound dressing. Carb. Pol., 69, 583-589. DOI: 10.1016/j.carbpol. 2007.01.014.

6. Dominici, M., Le Blanc, K., Meller, I., Slaper-Cortenbach, I., Marini, F. C., Krause, D. S., et al., 2006: Minimal criteria for defining mesenchymal stromal cells. The International Society for Cellular Therapy position statement. Cytotherapy, 8, 315-317.

7. Ehlers, E. M., Fuss, M., Rohwedel, J., Russlies, M., Kuehnel, W., Behrens, P., 1999: Development of a biocomposite to fill out articular cartilage lesions. Light, scanning and transmission electron microscopy of sheep chondrocytes cultured on a collagen I/III sponge. Ann. Anat., 181 (6), 513-518. DOI: 10.1016/S0940-9602(99)80055-7.

8. Fernandez Vallone, V. B., Romaniuk, M. A., Choi, H., Labovsky, V., Otaeugi, J., Chasseing, N. A., 2013: Review: Mesenchymal stem cells and their use in therapy: What has been achieved? Differentiation, 85 (1-2), 1-10.

9. Godbey, W. T., Hindy, B. S. S., Sherman, M. E., Atala, A., 2004: A novel use of centrifugal force for cell seeding into porous scaffolds. Biomaterials, 25, 2799-2805. DOI: 10.1016/j. biomaterials.2003.09.056.

10. Gogolewski, S., 2000: Bioresorbable polymers in trauma and bone surgery. Injury, 31, S-D28-32.

11. Griffon, D. J., Sedighi, M. R., Schaeffer, D. V., Eurell, J. A., Johnson, A. L., 2006: Chitosan scaffolds: Interconnective pore size and cartilage engineering. Acta Biomaterialia, 2 (3), $313-320$

12. Harvanova, D., Rosocha, Bakos, D., Švihla, R., Vasko, G., Hornak, S., et al., 2009: Collagen/hyaluronan membrane as a scaffold for chondrocyte cultivation. Biologia, 64 (5), 10321038. DOI: 10.2478/s11756-009-0171-y.

13. Chen, Y. L., Lee, H. P., Chan, H. Y., Sung, L. Y., Chen, H. CH., Hu, Y. CH., 2007: Composite chondroitin-6-sulfate/ dermatan sulfate/chitosan scaffolds for cartilage tissue engi- 
neering. Biomaterials, 28 (14), 2294-2305. DOI: 10.1016/j. biomaterials.2007.01.027.

14. Chen, G., Akahane, D., Kawazoe, N., Yamamoto, K., Tateishi, T., 2008: Chondrogenic differentiation of mesenchymal stem cells in a leakproof collagen sponge. Mat. Sci. Engin. C, 28 (1), 195-201. DOI: 10.1016/j.msec.2006.12.009.

15. Cho, J. H., Kim, S. H., Park, K. D., Jung, M. CH., Yang, W. I., Han, S. W., et al., 2004: Chondrogenic differentiation of human mesenchymal stem cells using a thermosensitive poly(N-isopropylacrylamide) and water-soluble chitosan copolymer. Biomaterials, 25 (26), 5743-5751. DOI: 10.1016/j. biomaterials.2004.01.051.

16. Johnstone, B., Hering, T. M., Caplan, A., Goldberg, V. M., Yoo, J. U., 1998: In vitro chondrogenesis of bone marrowderived mesenchymal progenitor cells. Exp. Cell Res., 238 (1), 265-272. DOI: 10.1006/excr.1997.3858.

17. Kessler, M. W., Grande, D. A, 2008: Review: Tissue engineering and cartilage. Organogenesis, 4 (1), 28-32. https:// doi.org/10.4161/org.6116.

18. Liu, C., Xia, Z., Czernuszka, J. T, 2007: Design and development of three-dimensional scaffolds for tissue engineering. Chem. Eng. Res. Design, 85(A7), 1051-1064.

19. Madihally, S. V., Matthew, H. W. T., 1999: Porous chitosan scaffolds for tissue engineering. Biomaterials, 20 (12), 11331142. DOI: 10.1016/S0142-9612(99)00011-3.

20. Malafaya, P. B., Silva, G. A., Reis, R. L., 2007: Natural-origin polymers as carriers and scaffolds for biomolecules and cell delivery in tissue engineering applications. Adv. Drug Del. Rev., 59 (4-5), 207-233. DOI:10.1016/j.addr.2007.03.012.

21. Medvecky, L., Giretova, M., Stulajterova, R., 2014: Properties and in vitro characterization of polyhydroxybutyratechitosan scaffolds prepared by modified precipitation method. J. Mat. Sci.: Mat. Med., 25 (3), 777-789. DOI:10.1007/ s10856-013-5105-0.

22. Mollon, B., Kandel, R., Chahai, J., Theodoropoulos, J., 2013: The clinical status of cartilage tissue regeneration in humans. Osteoarthritis and Cartilage, 21 (12), 1824-1833. DOI: 10.1016/j.joca.2013.08.024.

23. Muzzarelli, R. A. A., 2009: Review: Chitins and chitosans for the repair of wounded skin, nerve, cartilage and bone. Carb. Pol., 76 (2), 167-182. DOI: 10.1016/j.carbpol.2008.11.002.

24. Nair, S. L., Laurencin, C. T., 2007: Biodegradable polymers as biomaterials. Prog. Polym. Sci., 32, 762-798. http://dx.doi. org/10.1016/j.progpolymsci.2007.05.017.

25. Nehrer, S., Breinan, H. A., Ramappa, A., Young, G., Shortkroff, S., Louie, L. K., et al., 1997: Matrix collagen type and pore size influence behaviour of seeded canine chondrocytes. Biomaterials, 18 (11), 769-776.

26. Pelttari, K., Steck, E., Richter, W., 2008: The use of mesenchymal stem cells for chondrogenesis injury, Int. J. Care Inj., 39S1, S58-S65. DOI:10.1016/j.injury.2008.01.038.

27. Puppi, D., Chiellini, F., Piras, A. M., Chiellini, E., 2010: Polymeric materials for bone and cartilage repair. Progress in Polymer Science, 35, 403-444.

28. Solchaga, L. A., Penick, K. J., Welter, J. F., 2011: Chondrogenic differentiation of bone marrow-derived mesenchymal stem cells: Tips and tricks. Methods Mol. Biol., 698, 253-278. DOI: 10.1007/978-1-60761-999-4_20.

29. Tan, H., Chu, C.R., Payne, K. A., Marra, K. G., 2009: Injectable in situ forming biodegradable chitosan-hyaluronic acid based hydrogels for cartilage tissue engineering. Biomaterials, 30(13), 2499-2506. DOI: 10.1016/j.biomaterials.2008.12.080.

30. Tan, L., Ren, Y., Kuijer, R., 2012: A 1-min method for homogenous cell seeding in porous scaffolds. J. Biomat. Applic., 877-898. DOI: 10.1177/0885328210389504.

31. Tang, X., Fan, L., Pei, M., Zeng, L., Ge, Z., 2015: Evolving concepts of chondrogenic differentiation: history, state-ofthe-art and future perspectives. Eur. Cells Mater., 30, 12-27.

32. Vater, C., Kasten, P., Stiehler, M., 2011: Review. Culture media for the differentiation of mesenchymal stromal cells. Acta Biomaterialia, 7 (2), 463-477. DOI: 10.1016/j.actbio. 2010.07.037.

33. Wang, Y., Bian, Y. Z., Wu, Q., Chen, G. Q., 2008: Evaluation of three-dimensional scaffolds prepared from poly(3hydroxybutyrate-co-3-hydroxyhexanoate) for growth of allogeneic chondrocytes for cartilage repair in rabbits. Biomaterials, 29 (19), 2858-2868. DOI: 10.1016/j.biomaterials.2008.03.021.

34. Yamane, S., Iwasaki, N., Majima, T., Fukanoshi, T., Masuko, T., Harada, K., et al., 2005: Feasibility of chitosan-based hyaluronic acid hybrid biomaterial for a novel scaffold in cartilage tissue engineering. Biomaterials, 26 (6), 611-619. DOI: 10.1016/j.biomaterials.2004.03.013

35. Yang, B., Li, X. Y., Shi, S., Kong, X. Y., Guo, G., Huang, M. J., et al., 2010: Preparation and characterization of a novel chitosan scaffold. Carb. Pol., 80 (3), 860-865.

Received January 18, 2019

Accepted February 22, 2019 